\title{
Anatomía y Neuroanatomía, Disciplinas Perjudicadas por la Reforma Curricular
}

\author{
Anatomy and Neuroanatomy the Most Impair in the Curricular Reform
}

\author{
Oscar Inzunza; Alex Vargas \& Hermes Bravo
}

INZUNZA, O.; VARGAS, A. \& BRAVO, H. Anatomía y Neuroanatomía, disciplinas perjudicadas por la reforma curricular. Int. J. Morphol., 25(4):825-830, 2007.

RESUMEN: En los últimos tiempos, la docencia en Morfología ha debido enfrentar un escenario cambiante, producto de: a) un cambio curricular que ha llevado a la reducción de las horas destinadas a los cursos de Anatomía y Neuroanatomía; b) la dificultad creciente de obtener material cadavérico para las actividades prácticas de dichos cursos; c) el incremento en el número de alumnos de los cursos de pregrado; y, d) la reducción alarmante de los cultores de estas ciencias básicas del currículo de Medicina. Como resultado de lo anterior, es dable esperar, que esta situación -que tiene un alcance mundial-, más temprano que tarde tenga un efecto en el conocimiento morfológico de los médicos recién formados. En este estudio se analiza el rendimiento obtenido en pruebas diagnósticas de reconocimiento de estructuras en Anatomía y Neuroanatomía, aplicadas a médicos residentes de primer año de la especialidad de Radiología, formados bajo este nuevo paradigma curricular. Los resultados mostraron que la nota promedio (escala 1 a 7 ) de la prueba diagnóstica fue $3,14+$ 0,99. Es interesante notar que para una evaluación similar, los alumnos de primer año de la carrera de medicina obtienen una nota promedio de 5,75 + 0,55. Luego de aplicar a los mismos residentes del estudio un curso teórico-práctico de nivelación en Anatomía, se aprecia un mejoramiento en el reconocimiento de estructuras anatómicas, con nota promedio de 4,79+0,96; detectándose una mejora sustantiva en la identificación de elementos en secciones anatómicas de tronco. En las evaluaciones de temas neuroanatómicos, las diferencias son aún más notorias $(1,53 \mathrm{v} / \mathrm{s} 3,97)$. Estos datos reposicionan a las actividades prácticas de Anatomía, en especial el trabajo con material cadavérico, como un quehacer central en el proceso de aprendizaje de estos temas. Esta situación de olvido y/o desconocimiento observada en los nuevos médicos, ha generado una gran demanda por cursos de apoyo morfológico para las distintas especialidades.

PALABRAS CLAVE: Anatomía; Neuroanatomía; Docencia, Reconocimiento de estructuras.

\section{INTRODUCCION}

En la década de los años noventa, las ciencias morfológicas fueron seriamente afectadas por un proceso de reforma curricular de origen multicéntrico y de alcance global, que se verificó en Escuelas de Medicina americanas, europeas y asiáticas (McKeown et al., 2003; Plaisant et al., 2004, Leong, 1999). El efecto neto de este proceso fue la reducción notoria del creditaje y de las horas lectivas en los cursos de Anatomía, Neuroanatomía, Histología y Embriología (Drake et al., 2002; Inzunza et al., 2003).

Desde una perspectiva histórica, esta reforma curricular fue precedida por notables progresos en la Biología Molecular y en la Genética, desarrollados en los años setenta, que marcaron polos de desarrollo científico en estas áreas y que fue decididamente apoyado por las entidades de financiamiento, públicas y privadas; fomentándose así estas florecientes ciencias en desmedro de las ciencias morfológicas en general (Azíz et al., 2002). El cúmulo de conocimiento científico así generado, vino a incrementar la densidad del currículo de la Carrera de Medicina, generando presiones sobre los tiempos dedicados a otros ramos de los primeros años de Medicina (McCuskey et al., 2005).

El comienzo del siglo XXI se ha caracterizado por un avance tecnológico notable, con la emergencia de sofisticadas técnicas de imágenes médicas tridimensionales y el desarrollo de terapias mínimamente invasivas en el ámbito de la cardiocirugía, neurocirugía, etc., que, literalmente, han introducido en el ambiente médico una nueva forma de ver la anatomía (Paalman, 2000). Irónicamente, en el momento actual, en que el conocimiento anatómico está siendo intensamente requerido, las ciencias morfológicas en 
nuestro país parecen encontrarse en una situación inconfortable, caracterizada por: a) la reducción de las horas dedicadas a los ramos morfológicos, b) la disminución de los cultores de las ciencias morfológicas, c) el menguado acceso a material cadavérico, y, d) el incremento en el número de alumnos en relación al número de docentes por curso.

A fin de aquilatar la situación actual de la Anatomía en el medio local, en el presente trabajo se compara el rendimiento de los alumnos de primer año de Medicina con el rendimiento de los residentes de Radiología, provenientes de distintas Escuelas de Medicina, en pruebas prácticas de reconocimiento de estructuras en las áreas de Anatomía general y Neuroanatomía; evaluaciones realizadas tanto en preparaciones cadavéricas como en secciones corporales.

\section{MATERIAL Y MÉTODO}

Se comparan las notas obtenidas (con escala de 1 a 7) por alumnos de pregrado de Medicina y por médicos residentes de primer año de la especialidad de Radiología en pruebas prácticas de reconocimiento de elementos anatómicos. Estas evaluaciones, estructuradas como pruebas prácticas objetivadas de reconocimiento y aplicación del conocimiento (Inzunza et al., 2003), constaron de 21 estaciones, cada una con cuatro ítems a responder. En las estaciones se presentaron disecciones anatómicas de las diferentes regiones corporales y secciones transversales y sagitales de troncos humanos plastinados; todos estos elementos debidamente marcados (Fig. 1). Además, se presentaron cuatro fotos de disecciones anatómicas tratadas como animaciones (Inzunza y Bravo, 2002) y presentadas como imágenes computacionales (Fig. 2).

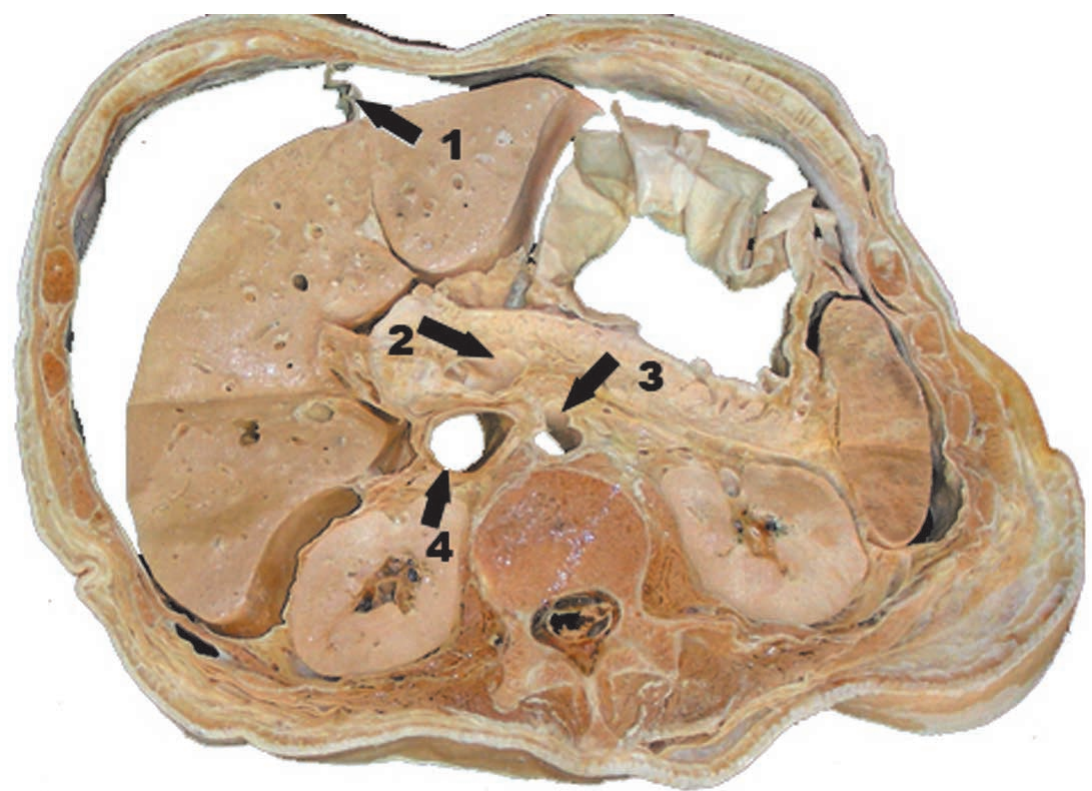

Fig. 1. Corte transversal de tronco, a nivel de L2; material plastinado, utilizado en las evaluaciones prácticas de los alumnos de pregrado y los becados de Radiología. Imágenes de este tipo están disponibles en nuestro "Portal de Anatomía Regional Humana" cuya dirección es: http://escuela.med.puc.cl/paginas/Departamentos/Anatomia/ PaginasWeb/Portada.html

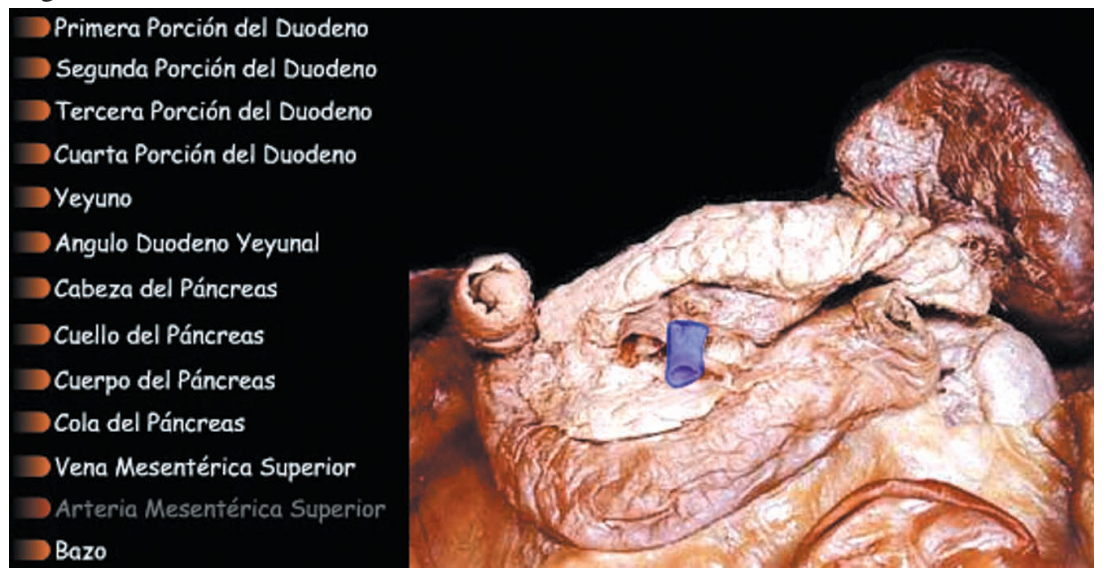

Fig. 2. Foto de una preparación anatómica tratada como animación computacional; imágenes como ésta, modificadas para preguntar, fueron utilizadas en las evaluaciones prácticas de los alumnos de pregrado y los becados de Radiología. Este material docente se encuentra disponible en nuestro "Portal de Anatomía Regional Humana" cuya dirección es: http://escuela.med.puc.cl/paginas/Departamentos/Anatomia/PaginasWeb/ Portada.html

Estas evaluaciones fueron aplicadas como examen final para el curso de pregrado "Anatomía y Embriología Humana MED 101A", año 2006 y como evaluación diagnóstica previa al curso "Anatomía para residentes de Radiología”, que imparte nuestro Departamento de Anatomía, años 2004 al 2006. Para los residentes, seis de las veintiuna estaciones de evaluación estaban dedicadas a temas de neuroanatomía, con preguntas en cortes y en preparaciones de encéfalo y médula espinal (Fig. 3). Estos resultados son analizados separadamente a fin de visualizar las diferencias en el rendimiento de cada grupo en dichos temas. Una evaluación de similares características fue realizada, a modo de examen, al final del curso de Anatomía para los residentes de Radiología, incluyendo preguntas de Anatomía y Neuroanatomía. 

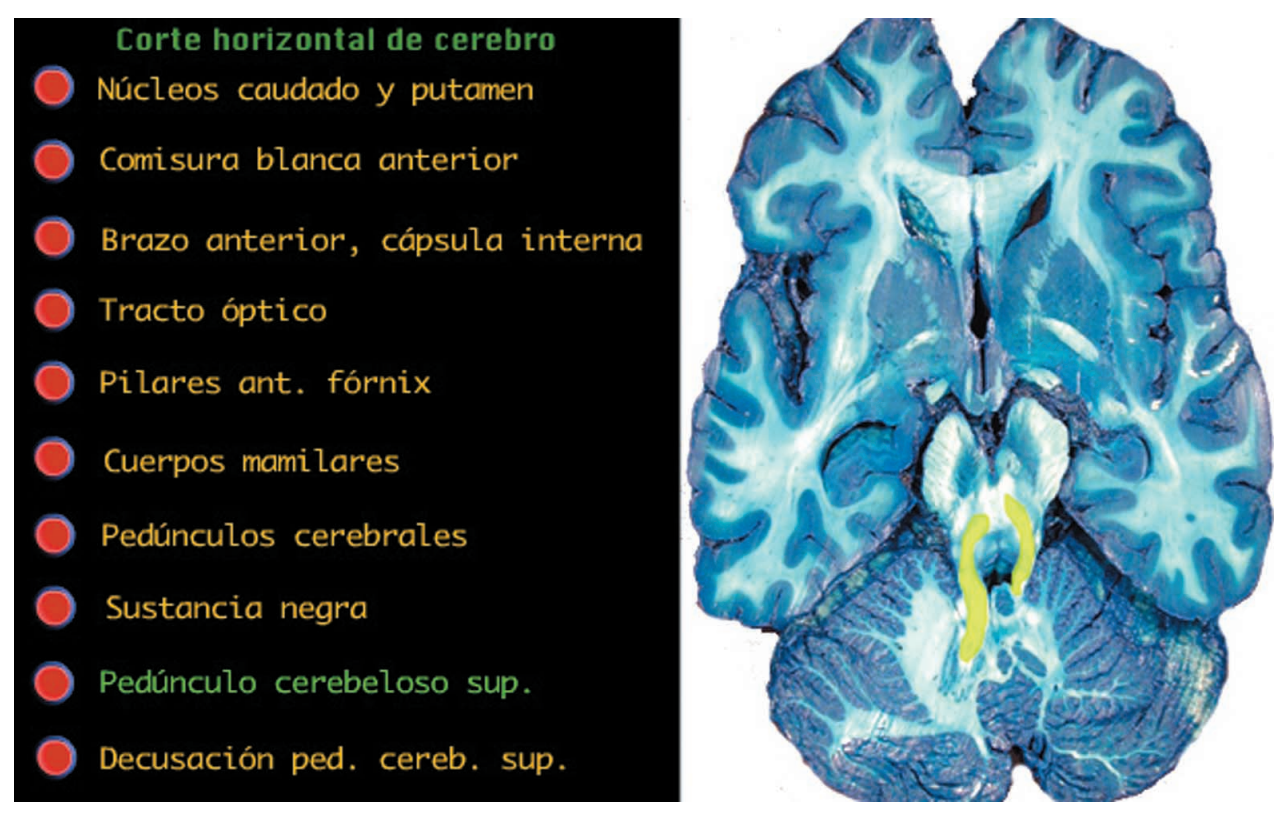

Fig. 3. Foto de animación con flash de corte horizontal de cerebro teñido con la técnica de Barnard, Roberts y Brown y posteriormente incluido en resina poliéster. Esta preparación modificada para preguntar, fue utilizada en las evaluaciones prácticas de los alumnos de pregrado y los becados de Radiología (Tomada de Bravo, H., Int. J. Morphol., 24(3):475480, 2006).

En atención al valor creciente que ha adquirido el diagnóstico por imágenes de tomografías y resonancias magnéticas, analizamos, separadamente, el rendimiento de los dos grupos estudiados: alumnos de pregrado $\mathrm{v} / \mathrm{s}$ residentes de Radiología, en las estaciones de evaluación donde se presentaban secciones transversales y sagitales de troncos humanos plastinados (Bravo, 2006).

\section{RESULTADOS}

El análisis comparativo de las notas obtenidas por los alumnos en el examen final de nuestro curso de pregrado "Anatomía y Embriología Humana MED 101", año 2006, versus los resultados de la evaluaciones aplicadas a los becados de Radiología, muestra claras diferencias. Los alumnos de pregrado (113 alumnos), obtienen en el examen final de Anatomía una nota promedio de 5,75 . Los becados de radiología consiguen en la evaluación diagnóstica referida a temas anatómicos una nota promedio de 3,14 ; valor que sube a 4,79 en el examen final del curso de Anatomía para los
Tabla I. Rendimiento en evaluaciones practicas. Comparación múltiple de pares, Método de Dunn.

\begin{tabular}{cccc}
\hline ExAls & v/s & Rxpre & si \\
ExAls & v/s & Rxpost & si \\
Rxpost & v/s & Rxpre & si \\
\hline
\end{tabular}

$\mathrm{p}<0,05$ residentes de Radiología (Fig. 4 y Tabla I). En relación con los resultados obtenidos en los temas neuroanatómicos, la evaluación diagnóstica arroja una nota promedio de 1,53;

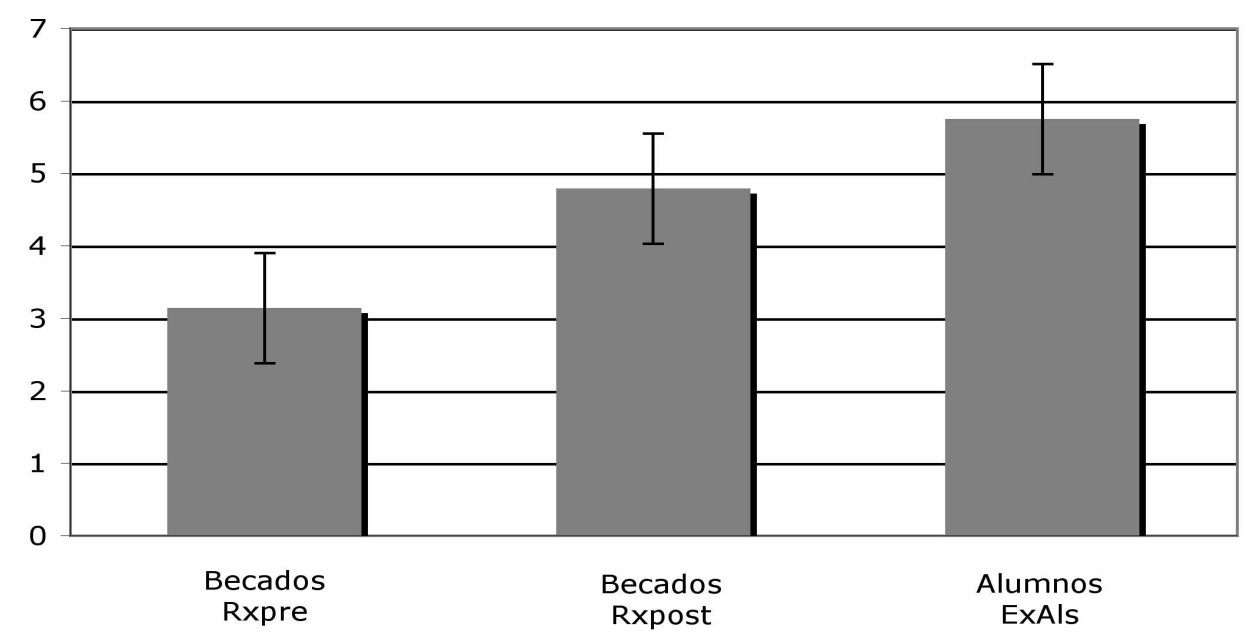

Fig. 4. Promedio de notas en evaluaciones prácticas. 
guarismo que sube a 3,97 en el examen final del curso de nivelación para los residentes de radiología. Al aplicar el análisis de varianza y la comparación múltiple de pares con el método de Dunn, se aprecia que las diferencias entre los valores señalados son estadísticamente significativas (Fig. 5 y Tabla II).

Al analizar por separado los resultados obtenidos por estos grupos en las estaciones de evaluación, donde se presentaban secciones transversales y sagitales de troncos humanos plastinados, se aprecian algunos datos particulares. Los alumnos de pregrado obtienen una nota promedio de 5,11 . Los becados alcanzan en la evaluación diagnóstica una nota promedio de 4,53; valor que asciende a 5,33 en el examen final de su curso de Anatomía, superando al promedio obtenido por los alumnos de pregrado (Fig. 3). Al aplicar el análisis de varianza y la comparación múltiple de pares con el método de Dunn, en las preguntas de anatomía seccional se aprecia una diferencia significativa entre los promedios obtenidos por los alumnos de pregrado, versus los resultados de la evaluación diagnóstica de los becados de Radiología. Del mismo modo, las diferencias entre las notas obteni-

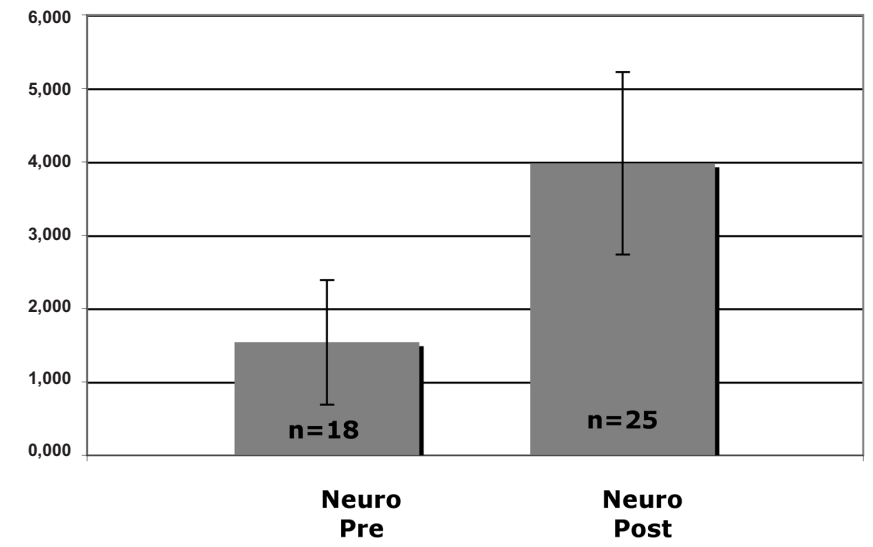

Fig. 5. Rendimiento de Becados en preguntas de Neuroanatomía

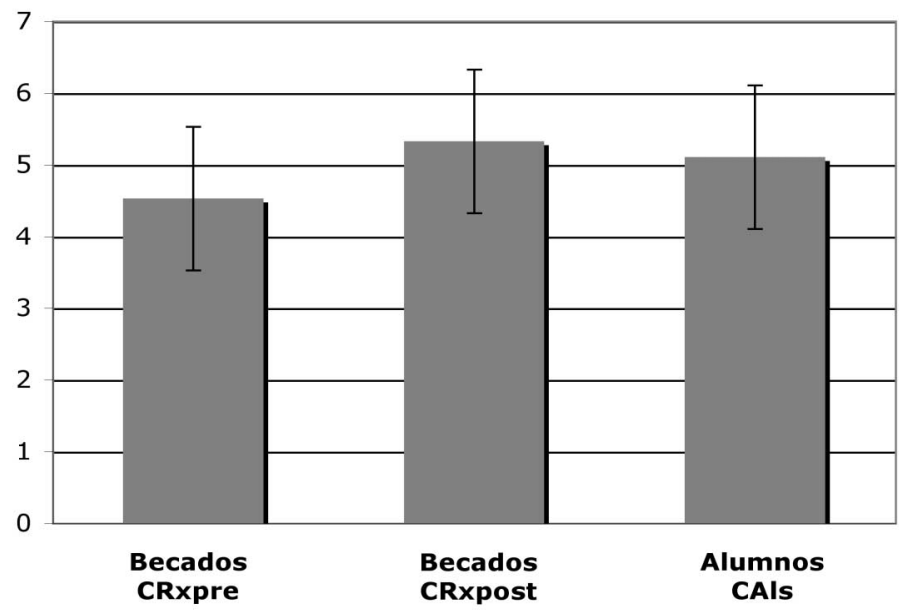

Fig. 6. Promedio de Notas en Anatomía Seccional.
Tabla II. Test de rango de la sumatoria, Mann Whitney. Rendimiento de becados en preguntas de Neuroanatomía.

\begin{tabular}{lcc}
\hline & Notas Pre & Notas Post \\
\hline Nota promedio & 1,538 & 3,977 \\
Mediana & 1,000 & 4,380 \\
$25 \%$ & 1,000 & 3,370 \\
$75 \%$ & 1,750 & 4,810 \\
\hline
\end{tabular}

$\mathrm{p}<0,01$

das, en este tipo de preguntas, por los becados de Radiología en la evaluación diagnóstica y en el examen final, son significativas; sin embargo, las diferencias entre las notas de los alumnos de pregrado y el examen de los becados no son significativas (Tabla III).

Tabla III. Rendimiento en Anatomía Seccional. Comparación Múltiple de Pares, Método de Dunn.

\begin{tabular}{lccc}
\hline CRxpost & v/s & CRxpre & si \\
CRxpost & v/s & CAls & no \\
CAls & v/s & CRxpre & si \\
\hline $\mathrm{p}<0,05$ & & &
\end{tabular}

\section{DISCUSIÓN}

En los últimos veinte años, hemos asistido a un proceso continuo de cambios en la docencia en los ramos básicos de Medicina; cambios generados por un lado, por el progreso notable de algunas áreas del conocimiento científico como la biología molecular y la genética (McCuskey et al.) y, por otro - talvez como consecuencia de lo anterior - por la necesaria e ineludible discusión de conceptos filosóficos y morales al interior de las Escuelas de Medicina, lo que se ha materializado en cursos de Ética y Bioética (Vial, 2004).

La necesidad de incorporar estos nuevos conocimientos al sobrecargado currículo, ha afectado especialmente a los ramos morfológicos, los cuales han visto seriamente interesadas sus horas lectivas. Así, en el ámbito nacional, prácticamente todos los cursos de Anatomía, Neuroanatomía, Embriología e Histología han visto mermadas sus horas docentes; a esto es necesario agregar el incremento en el número de alumnos por curso, los cuales efectivamente han doblado su matrícula. Irónicamente, estos procesos de ajuste y reducción se desarrollan en paralelo con los progresos tecnológicos en el campo de la imagenología médica, área de continuo y sostenido crecimiento que está demandando de manera importante a la Anatomía Humana in vivo (Paalman, 2000). 
Nuestros datos detectaron un inadecuado rendimiento en la identificación de estructuras anatómicas, por parte de los residentes de primer año de Radiología en la evaluación diagnóstica, situándose muy por debajo del rendimiento del curso de pregrado $(3,14 \mathrm{v} / \mathrm{s} 5,75)$. Esto puede ser explicado por el "factor olvido", considerando que, en prácticamente todas las Escuelas de Medicina, los cursos de anatomía se ubican en el primer año de la Carrera, momento único en el cual es posible realizar un estudio detenido, mediante disecciones o prosecciones, del cuerpo humano. La situación es en extremo deplorable para los temas neuroanatómicos donde, en la evaluación diagnóstica, la nota promedio de los residentes es de 1,53. Aquí, además del "factor olvido", inciden otros factores; como el hecho de que estos temas complejos son tratados en tiempos muy ajustados, generalmente desvinculados de la aplicación clínica, utilizando poco trabajo de laboratorio para su aprendizaje (Drake et al., 2002).

Los datos mostraron que con el curso teórico-práctico de Anatomía para becados de Radiología, curso de 16 sesiones (12 de Anatomía y 4 de Neuroanatomía), de 2 horas cada una, se logra mejorar en forma importante el rendimiento de los becados, no alcanzando eso sí el nivel de los alumnos de pregrado $(4,79 \mathrm{v} / \mathrm{s} 5,75)$. Esto demuestra que "olvidar un tema determinado, no es lo mismo que no saber del tema en cuestión".

Llama la atención la mejoría notable en el rendimiento en los temas neuroanatómicos $(1,53 \mathrm{v} / \mathrm{s} 3,97)$. Esta situación que puede ser explicada, por un lado, por el carácter eminentemente práctico del curso de nivelación y, por otro, por la familiarización de los becados con las preparaciones neuroanatómicas, considerando el entrenamiento preferentemente visual a que son sometidos los residentes de Radiología; ya que la morfología en general -considerando a la Radiología como otro modo de estudiar la forma- y la anatomía en particular, corresponden a ciencias donde las claves visuales son relevantes para su aprendizaje (Bravo \& Inzunza, 1995).

Estos becados, la mayoría de ellos médicos recién egresados de alta calificación, tienen en común una formación en anatomía, marcada por restricciones de tiempo y de acceso al material cadavérico. Una situación similar hemos detectado en un estudio en progreso, en términos de rendimiento en pruebas de reconocimiento de estructuras anatómicas en los residentes de primer año de traumatología.

Es interesante notar, en la evaluación diagnóstica de los becados de radiología, que el rendimiento en la identificación de estructuras en secciones corporales es superior al promedio obtenido por ellos en esa misma evaluación $(4,53 \mathrm{v} / \mathrm{s} 3,14)$; situación que puede ser explicada por la capacidad de estos noveles médicos de extrapolar desde la experiencia en imagenología médica a la preparación anatómica. Así y todo, el rendimiento de los becados en la prueba de diagnóstico, en este tipo de preguntas, es menor a los resultados obtenidos por los alumnos de pregrado $(4,53 \mathrm{v} / \mathrm{s} 5,11)$; situación que no nos sorprende ya que nuestros alumnos son constantemente requeridos en el estudio de la anatomía seccional (Inzunza et al., 2003). Al final del curso "Anatomía para becados de Radiología" se hace evidente en ellos una notable mejoría en la capacidad de identificar estructuras anatómicas en secciones corporales, superando al curso de pregrado en este tipo de preguntas $(5,33 \mathrm{v} / \mathrm{s} 5,11)$, hecho que puede explicarse por el énfasis práctico de este curso de anatomía como también por la experiencia ganada concomitantemente por los becados en estudios resonancias y tomografías seccionales, situación que tiene un significativo impacto en la identificación de estructuras anatómicas (de Barros et al., 2001). Esto también puede dar cuenta de las diferencias observadas en los alumnos de pregrado entre los valores obtenidos en el examen final y las preguntas de anatomía seccional $(5,75 \mathrm{v} / \mathrm{s} 5,11)$ corroborando lo señalado por de Barros et al.

Estos antecedentes reposicionan a las actividades prácticas de anatomía, en especial el trabajo con material cadavérico, como un quehacer central en el proceso de aprendizaje de estos temas. Esta situación de olvido observada en los noveles médicos, ha generado una gran demanda por cursos de apoyo morfológico para las distintas especialidades en nuestra Escuela de Medicina (ver página web: http://escuela.med.puc.cl/paginas/Departamentos/Anatomia/Anatomia_Post.html, situación que parece generalizarse en el país.

INZUNZA, O.; VARGAS,A.\& BRAVO,H. Anatomy and Neuroanatomy, the most impair in the curricular reform. Int. J. Morphol., 25(4):825-830, 2007.

SUMMARY: In the last years morphology teaching has being influenced due to a changing environment which includes a) Reduction in the number of credits in the courses of anatomy and neuroanatomy; b) The increasing difficulty to obtain cadaveric material for teaching purposes; c) The increase in the number of students; and d) The reduction of the numbers of instructors and professors in morphological sciences. As a consequence of these changes there is impairment in the knowlegde of this basic courses in the present medical graduates. In this paper we analyzed the anatomical knowledge obtained in practical test applied to residents in radiology. The results show that the average grade (scale 1 to 7 ) gets in the diagnostic tests is $3,14+0,99$. It is interesting to point out that a similar test apply to undergraduate medical students of the first year, shows an average grade of $5,75+0,55$. After a theoretical and a practical course given to the residents mentioned before, these postgraduate students were examined again. The average grade obtained this time was 4,79+0,96, showing an important increment in the anatomical knowledge. In the neuroanatomical questions, the performance previous and posterior to the course was much better $(1,53$ $\mathrm{v} / \mathrm{s} 3,97)$. These results point out to the importance of the practical teaching activities with cadaveric material as a core process in the anatomical learning. The issue of forgotten antomical knowledge in the recent graduated residents has generated an increased request of anatomical practical courses for different medical specialties.

KEY WORDS:Anatomy; Neuroanatomy; Teaching 


\section{REFERENCIAS BIBLIOGRÁFICAS}

Azíz, MA.; McKenczie, J. C.; Wilson, J. S.; Cowie, R. J.; Ayeni, S. A. \& Dunn, B. K. The Human cadaver in the age of biomedical informatics. Anat. Rec., 269:20$32,2002$.

Bravo H. Plastinación, una herramienta adicional para la Eenseñanza de la Anatomía. Int. J. Morphol., 24(3)475-80, 2006.

Bravo, H. \& Inzunza, O. Evaluación de algunos programas computacionales en la enseñanza de la Anatomía y Neuroanatomía de la Facultad de Medicina de la Pontificia Universidad Católica de Chile. Rev. Chil. Anat., 13(1):79-86, 1995.

de Barros, N.; Rodrigues, C. J.; Rodrigues Jr., A. J.; de Negri Germano, M. A. \& Cerri, G. G. The value of teaching sectional anatomy to improve CT scan interpretation. Clin. Anat., 14: 36-41, 2001.

Drake, R. L.; Lowrie, D. J. \& Prewitt, C. M. Survey of Gross Anatomy, Microscopic Anatomy, Neuroscience, and Embryology courses in Medical School curricula in the United States. Anat. Rec., 269:118-22, 2002.

Inzunza, O; D’Acuña, E. \& Bravo, H. Evaluación práctica deAnatomía. Rendimiento de los alumnos de primer año de Medicina ante distintas formas de preguntar. Int. J. Morphol 21(2):131-6, 2003.

Inzunza, O. \& Bravo, H. Animación computacional de fotografías, un real aporte al aprendizaje práctico de Anatomía Humana. Rev. Chil. Anat., 20(2):151, 2002.

Leong, S. K. Back to basics. Clin.Anat.,12(6):422-6, 1999.

McCuskey, R.; Stephen, W.; Camichael, \& Darrell G. Kirch. The importance of Anatomy in health professions education and the shortage of qualified educators. Academic Medicine, 80(4):349-51, 2005.

McKeown, P. P.; Heylings, D. J.; Stevenson, M.; McKelvey, K. J.; Nixon, J. R. \& McCluskey, D. R. The impact of curricular change on medical students' knowledge of anatomy. Med. Educ., 37(11):954-61, 2003.

Paalman, M. H. Why teach anatomy? Anatomists respond. Anat. Rec., (New Anat) 261:1-2, 2000.

Plaisant, O.; Cabanis, E. A. \& Delmas, V.Going back to dissection in a medical currículo: the paradigm of Necker-Enfants Malades. Surg. Radiol. Anat., 26:504$11,2004$.

Vial, J. de D. Ética en la formación médica. Ars Médica, 9:122-9, 2004.

Dirección para correspondencia:

Prof. Dr. Oscar Inzunza

Departamento de Anatomía Normal

Escuela de Medicina,

Pontificia Universidad Católica de Chile

CHILE

Teléfono 56-2-354 3076

Email: oinzunza@med.puc.cl

Recibido : 22-06-2007

Aceptado: 18-10-2007 